\title{
The use of kerogen data in understanding the properties and evolution of interstellar carbonaceous dust
}

\author{
R. Papoular*
}

\author{
Sce Chimie Moléculaire, CEA Saclay, France
}

Received 27 June 2001 / Accepted 29 August 2001

\begin{abstract}
A number of authors have, in the past decade, pointed to the similarity of the 3.4- $\mu \mathrm{m}$ band of kerogen with that of the Galactic Centre (GC). Kerogen is a family of solid terrestrial sedimentary materials essentially made of $\mathrm{C}, \mathrm{H}$ and $\mathrm{O}$ interlocked in a disordered, more or less aliphatic, structure. Here, the most recent results of the astronomical literature and the rich quantitative geochemical literature are tapped with two purposes in mind: extend the analogy to the mid-IR bands and, based on these new constraints, quantitatively assess the properties of the carrier dust. It is shown that the great diversity of IR astronomical IS (interstellar) dust is paralleled by the changes in kerogen spectra as the material spontaneously and continuously evolves (aromatizes) in the earth. Since the composition and structure of kerogen are known all along its evolution, it is possible, by spectral analogy, to estimate these properties for the corresponding astronomical carriers. The Galactic Centre $3.4 \mu \mathrm{m}$ feature is thus found to correspond to an early stage of evolution, for which the composition in $\mathrm{C}, \mathrm{H}$ and $\mathrm{O}$ and the structure of the corresponding kerogen are known and reported here. The role of oxygen in the subsequent evolution and its contribution to different bands are stressed. The above provides new arguments in favour of the 3.4- $\mu \mathrm{m}$ band, as well as the observed accompanying mid-IR bands, being carried by kerogen-like dust born in CS (circumstellar) envelopes, mostly of AGB (asymptotic giant branch) objects. Subsequent dust evolution in composition and structure (aromatization) is fast enough that the unidentified infrared bands can already show up in well-developed planetary nebulae (PNe), as observed. A fraction of incompletely evolved dust can escape into the diffuse IS medium and molecular clouds. As a consequence, aliphatic and aromatic features can both be detected in the sky, in emission (Proto-PNe, PNe and PDRs (photo-dissociation regions)) as well as in absorption (dense molecular clouds and diffuse ISM). Changes in wavelength and band width with line of sight are explained by changes in the nature and number of chemical functional groups composing the carrier material. Predictions of the kerogen model in the UV and far IR are proposed for testing.
\end{abstract}

Key words. carbonaceous dust - kerogen - galactic centre - interstellar medium-dust birth and evolution

\section{Introduction}

A coherent trend of evolution in the IR spectra of carbonaceous dust has emerged in recent years (see Kwok et al. 1999; Guillois et al. 1996; Papoular et al. 1996; Buss et al. 1993, 1990 and references therein). The wealth of far IR spectra collected by the ISO satellite with good resolution and accuracy seems to confirm the conjecture that much of this evolution already takes place between the post-AGB and planetary-nebular stages of stellar life (Volk et al. 2001). On the other hand, terrestrial kerogens have comparable spectra, which undergo similar changes upon ageing or heating. It is the purpose of the present work to show that the parallel between available IR spectra of stellar dust and kerogen at successive stages of

\footnotetext{
* e-mail: papoular@wanadoo.fr
}

evolution makes it possible to approximately infer the composition and structure of the former, using the quantitative experimental data related to the corresponding properties of the latter.

It has long been known that the dominant constituent of the organic matter in meteoritic carbonaceous chondrites is similar to kerogen, which is the essential constituent of sedimentary rocks on Earth (see Kerridge 1990, p. 10). Kerogen is a family of highly disordered macromolecular organic materials made of $\mathrm{C}, \mathrm{H}$ and $\mathrm{O}$, and traces of $\mathrm{N}$ and $\mathrm{S}$ (hence the designation "CHON materials"). Similar materials, dubbed "CHON particles", were also discovered in comets (see Kissel et al. 1986). On the other hand, Pendleton et al. (1994) have produced an arresting fit of the 3.4- $\mu$ band observed towards the Galactic Centre with that of the organic extract of the Murchison meteorite, suggesting that kerogen might be a model of 
IS dust. This possibility had already been considered by Ehrenfreund et al. (1991), who looked for similarities between the spectra of meteorites and the GC, and those of several kerogens with different degrees of hydrogenation; unexpectedly, they concluded negatively, although one of their samples came quite close to the Murchison meteorite.

The idea again surfaced in a paper by Tielens et al. (1996) but was apparently abandoned in favour of hydrogenated amorphous carbon HAC in Chiar et al. (2000). However, based on mid-IR spectra of the GC region, these authors find it necessary to include in their model some oxygen impurities, of which HAC is devoid in principle. One demonstration of the critical role of oxygen can be found in Sakata et al. (1987), by comparing the IR absorption spectra of oxidized vs. non-oxidized (as deposited) QCC (quenched carbonaceous compounds), a form of hydrogenated amorphous carbons, in their Fig. 1.

One notes that, in the works cited above, the presence of oxygen in kerogens was overlooked although the properties of these materials and, hence, their IR spectra are largely dependent on this element (see below). Neither is mention made of the parallel variety (similar evolution in time?) of kerogen and CS and IS spectra. Also, the explicit comparisons between kerogen and GC spectra were limited to the $\mathrm{C}-\mathrm{H}$ stretch band $(3.4 \mu \mathrm{m})$, in which oxygen is not involved.

Whilst the carrier of the 3.4- $\mu$ feature has long been thought to be produced in dense molecular clouds in the form of refractory organic mantles over silicate cores (see Sandford et al. 1991; Greenberg 1978), this model is brought into question by the recent finding that, unlike the 9.7- $\mu \mathrm{m}$ feature, the 3.4- $\mu \mathrm{m}$ feature towards Sgr A* (IRS 7) is not polarized as expected (Adamson et al. 1999). Also, $A_{\mathrm{v}} / \tau(3.4)=150$ towards the GC, as compared with 270 , on average, for local ISM sources of various extinctions (Pendleton et al. 1994). This together with the spectral similarity of interstellar dust towards the GC and circumstellar dust around the PPN CRL 618 (Lequeux \& de Muizon 1990; Chiar et al. 1998) also calls for a reassessment of the origin and fate of organic dust in space.

Finally, although Papoular et al. (1996) broached the kerogen case in their comparison of solid-state carbonaceous models, they did not dwell on spectral analogies with galactic dust.

The present work is an attempt to fill in some of these gaps by making use of the wealth of spectral, chemical and physical data in the kerogen literature. The availability of measured integrated absorbances for very different members of this family and for all the conspicuous IR features will alleviate the well known difficulty in the way of estimating celestial abundances of particular functional groups, which is due to the fact that the specific IR crosssection of a group depends on its environment, i.e. on the particular composition and structure of the material.

\section{The properties of kerogen}

Kerogen is a solid sedimentary, insoluble, organic material found in the upper crust of the Earth in dispersed form (see Durand 1980). Its properties and spontaneous evolution in time are best understood by representing each sample in the 2-D van Krevelen diagram (Fig. 1), by a point $(\mathrm{P})$ whose abscissa and ordinate are respectively the ratio of $\mathrm{O}$ to $\mathrm{C}$ concentrations, and of $\mathrm{H}$ to $\mathrm{C}$ concentrations (all in number of atoms). The shallowest samples lie in the upper right side of this diagram; they are mainly composed of long aliphatic carbon chains. As time goes by, the samples are buried deeper and deeper, and their composition changes in an orderly manner: first, in the "diagenesis" phase, mostly oxygen is expelled in the form of $\mathrm{CO}, \mathrm{CO} 2$ and $\mathrm{H} 2 \mathrm{O}$ and the representative point shifts nearly horizontally to the left. Then, in "catagenesis", it rolls down towards the origin as mostly $\mathrm{H}$ is expelled in the form of methane, a process which breaks the aliphatic chains and allows aromatic rings to form and coalesce in clusters, which are thermodynamically more stable (see left side of figure).

This clear relationship between composition and structure explains why the location in the van Krevelen diagram roughly defines the properties of the material and, in particular, its IR spectrum. The initial properties of kerogen depend on its geographical location, so the samples are roughly classified as of lake (I), marine (II) and continental (III) origins, in order of decreasing initial $\mathrm{H}$ content. In Fig. 1, the corresponding evolutionary tracks lie in three roughly horizontal strips which, at low $O / C$ values, coalesce like branches of a tree into one nearly vertical stem extending towards the origin (see, for instance, Durand 1980, p. 129). In this diagram, which was originally proposed for coals (van Krevelen 1993), the latter are scattered, according to mining depth (i.e. evolutionary stage), nearly along the same bent strip as kerogens of type III. This is illustrated graphically in Durand (1980) and Papoular et al. (1996), Fig. 4, and is not duplicated here, in Fig. 1, for the sake of clarity. The main difference between kerogens and coals is that the latter are found in the form of bulk rocks and the former in dispersed form (sand-like).

The important point for present purposes is that hundreds of kerogen samples have been analyzed thoroughly, using all available analytical techniques: thermal, thermogravimetric, IR , Raman and NMR spectroscopy, etc. To each sample corresponds a point in the van Krevelen diagram, a complete chemical composition and, in many cases, an IR absorbance spectrum with integrated absorbances given for the main bands (example in Fig. 2). This made it possible to find correlations between bands and assign each band to a chemical functional group.

Moreover, the natural evolution (and the attendant displacement along the corresponding strip in the van Krevelen diagram) could be mimicked by annealing "young" samples (from shallow mines) up to a few hundred degrees Celcius. This shows that mild heating 


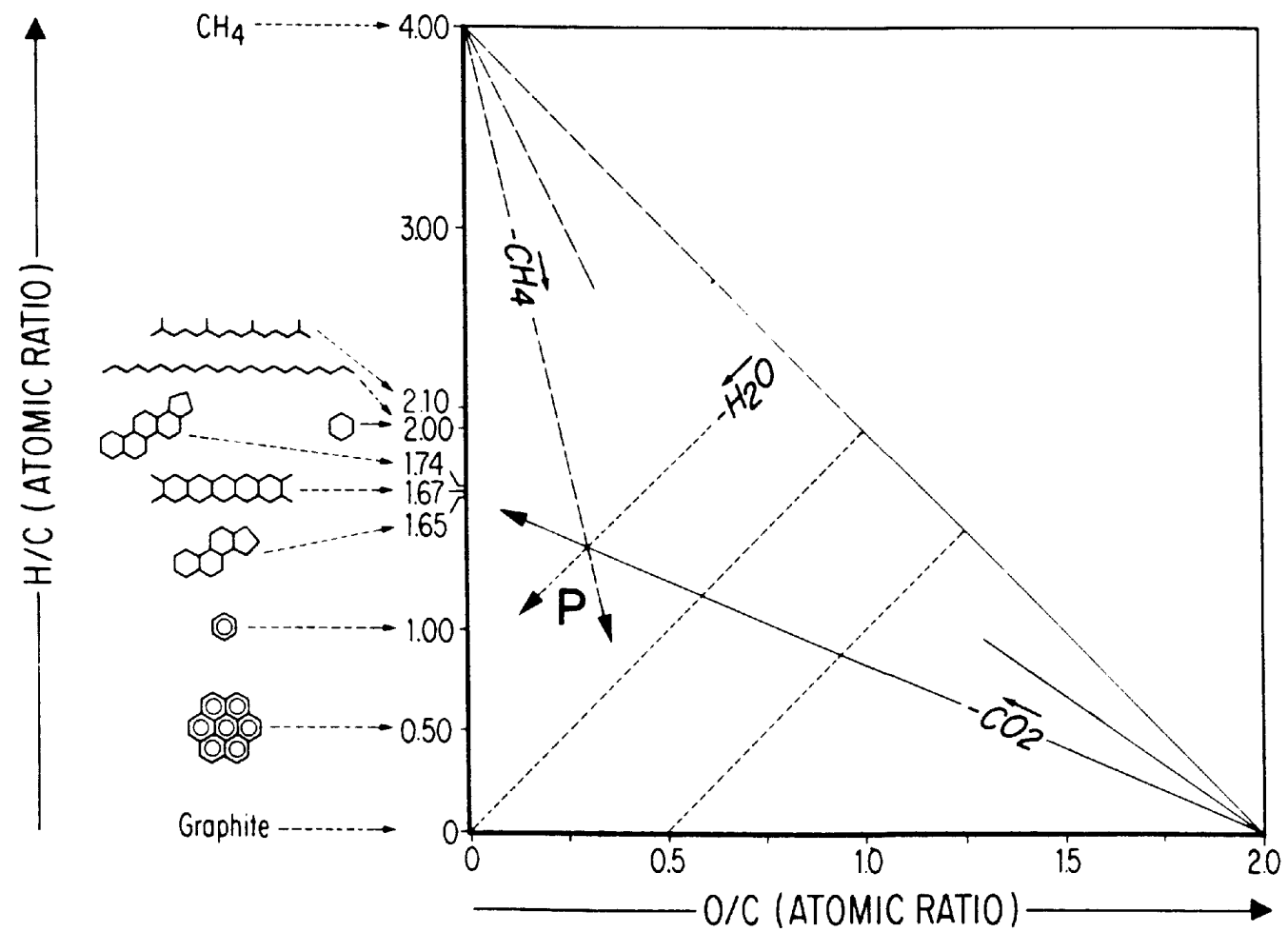

Fig. 1. Van Krevelen diagram (after Durand 1980, p. 122). Each arrow represents the direction of loss of a particular gas molecule in the course of evolution. A rough sketch of the main structural elements is given on the left side for a few evolutionary stages.

accelerates evolution in the natural (spontaneous) direction, an important hint to the evolutionary processes in space.

All these efforts culminated in the building up of a chemical representation of the structure of kerogens as a function of their type and evolutionary state, i.e. their location in the diagram of Fig. 1. The work of Behar \& Vandenbroucke (1986) is a good example of this development. Inspired by theoretical models of coal, they found that a disordered array of at least 1500 atoms of C, H, O, $\mathrm{N}$ and $\mathrm{S}$ in different configurations are necessary to describe the properties of a given kerogen. It is not possible to adequately describe the observed spontaneous and continuous changes in properties in terms of a limited number of small, specific, molecules. In order to fit the measurements on different samples, one has rather to build a random array made of a dominant carbon skeleton with functional groups of heteroatoms $(\mathrm{H}, \mathrm{O}, \mathrm{N}, \mathrm{S})$ attached randomly to it, then statistically tailor the number of different bondings $(\mathrm{C}-\mathrm{C}, \mathrm{C}=\mathrm{C}$, etc.), functional groups $(\mathrm{C}-\mathrm{H}$, $\mathrm{C}=\mathrm{O}$, etc.) and aromatic or polyaromatic rings, etc. The variety of environments of the functional groups ensures that their characteristic vibrations will blend into bands of the right width. The relative intensities of the latter will change according to the concentrations of the corresponding functional groups. This scheme accounts for the continuous set of IR spectra observed along the evolutionary track.

Behar \& Vandenbroucke give numbers for the structural parameters enumerated above and for 8 representative evolutionary stages. They also give sketches of the corresponding structures, an example of which will be shown and discussed below (Fig. 4).

\section{The IR spectrum}

As a typical example, Fig. 2 (adapted from Robin \& Rouxhet 1976) displays the IR absorbance spectrum of a young kerogen of type II $(H / C=1.32, O / C=0.104)$. From Robin et al. (1975), their peak wavenumber $\left(\mathrm{cm}^{-1}\right)$ and wavelength $(\mu \mathrm{m})$, integrated intensity $(\mathrm{cm} / \mathrm{mg})$ and assignment are

1. $\sim 3400(\sim 2.95) ; 46.2 ; \mathrm{OH}$ stretch; due partly to chemically bonded $\mathrm{OH}$ groups and partly to adsorbed or trapped, not chemically bonded, $\mathrm{H}_{2} \mathrm{O}$ molecules; the latter is responsible for the long redward tail, through $\mathrm{H}-$ bondings with other parts of the skeleton; peak position and band profile change considerably with $\mathrm{H}_{2} \mathrm{O}$ content (depending on evolutionary stage or heat treatment); see Robin \& Rouxhet (1976) and Unsworth et al. (1988). This feature is clearly distinct from the water ice band, which peaks near $3.1 \mu \mathrm{m}$ and has a much steeper red wing (associated to that observed in the sky towards young stellar objects (Gibb et al. 2000).

2. 3060 (3.27); C-H aromatic or olefinic stretch, barely visible here, and only measurable for very small $H / C$ and $O / C$ ratios.

3. 2920 (3.42); 48.2; blend of anti-symmetric and symmetric $\mathrm{CH}_{3}$ stretch at 2962 and 2872, asymmetric and symmetric $\mathrm{CH} 2$ stretch at 2926 and 2853; $\mathrm{CH}$ stretch at $2890 \mathrm{~cm}^{-1}$, respectively.

4. 1710 (5.85); 14; $\mathrm{C}=\mathrm{O}$ (ketone) stretch. 


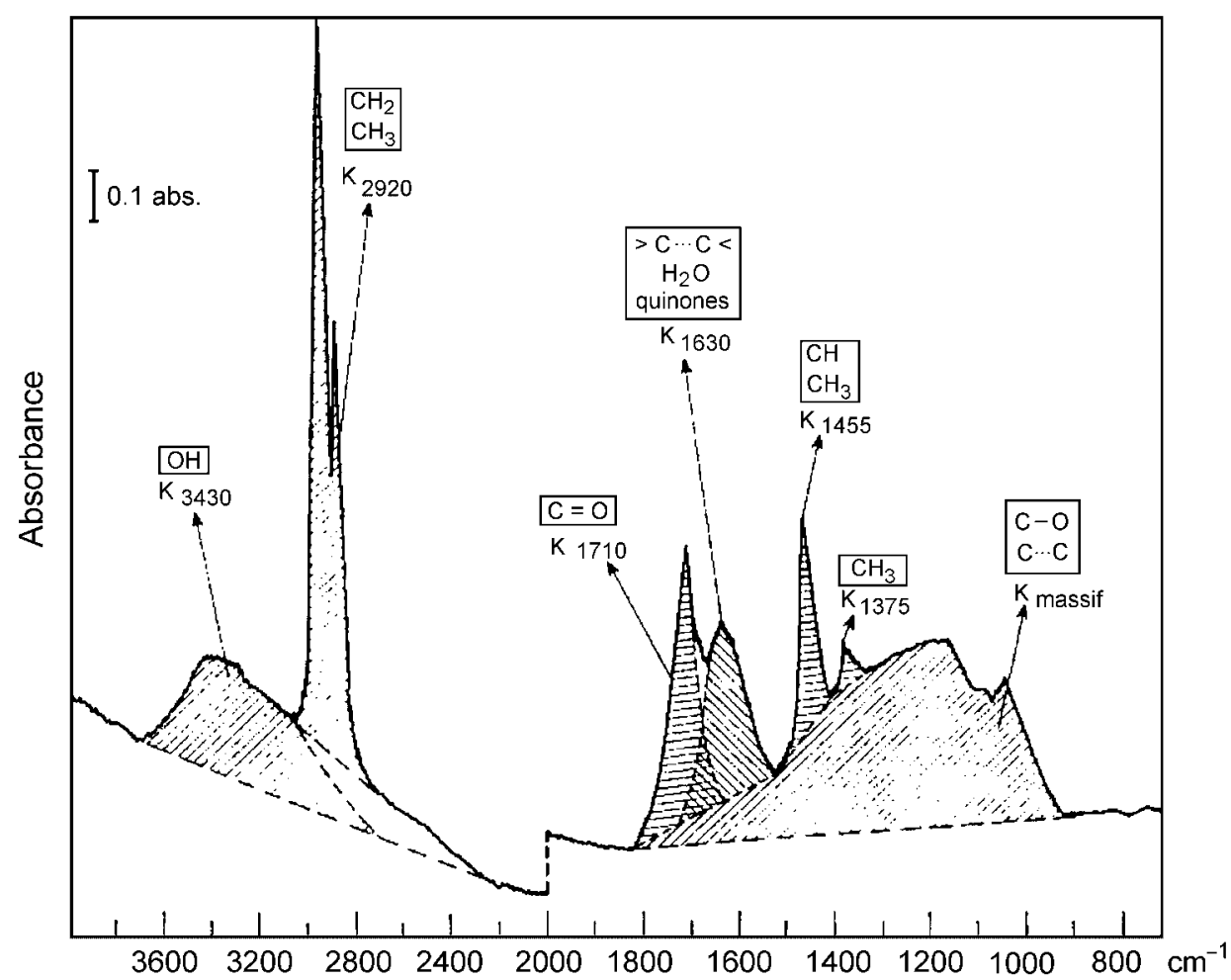

Fig. 2. Typical IR absorbance spectrum of a type II kerogen, after Robin \& Rouxhet (1976). The dashed areas are used to determine the band intensities, $K$ (see Eq. (1)). A number of partial assignments of bands are enclosed in squares; see details in Sect. 3.

5. $1630(6.15) ; 11.4$; disputed (but perhaps concurrent) assignments to $\mathrm{H}_{2} \mathrm{O}$ deformation, quinonic $\mathrm{C}=\mathrm{O}$ with $\mathrm{H}$ bond and $\mathrm{C}=\mathrm{C}$ olefinic and aromatic stretch (also see Painter et al. 1981).

6. 1455 (6.87); 6.1; asymmetric $\mathrm{CH}_{2}$ and $\mathrm{CH}_{3}$ deformation.

7. 1375 (7.28); 1.2; symmetric $\mathrm{CH}_{3}$ deformation.

8. 1800 to 900 (5.5 to 11); 67.3; massif, or plateau (underlying broad band) peaking near $8 \mu \mathrm{m}$, due to C...C and $\mathrm{C}-\mathrm{O}$ stretch, $\mathrm{C}-\mathrm{H}$ in-plane bend and $\mathrm{OH}$ deformation (see Durand 1980).

9. 930 to 700 (11 to 14 ); aromatic out-of-plane bending, depending on the number of adjacent protons; barely visible here, and never exceeds an intensity of 5 .

The intensity, $K$, given here (third number) is related to the absorbance, $\alpha$, by

$\alpha\left(\mathrm{cm}^{-1}\right)=2.3 \times 10^{3} \rho K / \Delta \nu$,

where $\rho$ is the material density in $\mathrm{g} \mathrm{cm}^{-3}(\sim 1)$ and $\Delta \nu$, the bandwidth in $\mathrm{cm}^{-1} . K(\mathrm{~cm} / \mathrm{mg})$ is $10^{-3}$ times the intensity usually defined in the astrophysical literature; it is also related to the integrated cross-section per $\mathrm{C}$ atom, $\Sigma$, by

$$
\begin{aligned}
& \Sigma\left(\mathrm{cm}^{2} \mathrm{~cm}^{-1} \mathrm{Catom}^{-1}\right)=4.7 \times 10^{-20} \\
& \quad \times\left(1+\frac{H}{12 C}+\frac{16 O}{12 C}\right) K \approx 4.7 \text { to } 7 \times 10^{-20} \mathrm{~K} .
\end{aligned}
$$

As expected, when referred to the corresponding functional group, $\Sigma$ turns out to be of the same order of magnitude as the corresponding average values, when available, given by Wexler (1968, p. 88-89), based on measurements of a large number of small molecules.

Not unexpectedly, a strong relationship holds between the bands at 2920 and $1455 \mathrm{~cm}^{-1}$ for all kerogen types and evolutionary stages (Robin \& Rouxhet 1976): the ratio of their integrated intensities is $\sim 8$. Also, $K(3.4 \mu \mathrm{m})$ increases linearly with $H / C$, from 0 at $\leq 0.3$ at least up to $80 \mathrm{~cm} / \mathrm{mg}$ at $H / C=1.3$.

\section{A model for the carrier of the GC 3.4- $\mu \mathrm{m}$ feature}

Let us now compare these bands with those which were observed towards the GC (see, e.g., Pendleton et al. 1994; Tielens et al. 1996; Chiar et al. 2000).

Depending on the IRS number (background star observed), the peak of the $\mathrm{OH}$ band near $3 \mu \mathrm{m}$ shifts from $\sim 3500$ to $\sim 3300 \mathrm{~cm}^{-1}$ and has a very long red tail. Waterice coatings are certainly deposited on carbon dust in cold environments, and are responsible for most of the observed $3.1-\mu \mathrm{m}$ peak. However they can hardly account for the lingering red wing, unless they are much thicker than $0.1 \mu \mathrm{m}$ (see Leger et al. 1983; Gibb et al. 2000). This has led Tielens et al. (1996) to wonder if the red tail was not due to an independent dust component, and Wada et al. (1991) to suggest $\mathrm{H}_{2} \mathrm{O}$ trapped in $\mathrm{SiO}$. Now, kerogen, too, contains such water of hydration which is responsible for its $3-\mu \mathrm{m}$ band (see Sect. 3.1) and the latter is quite similar 

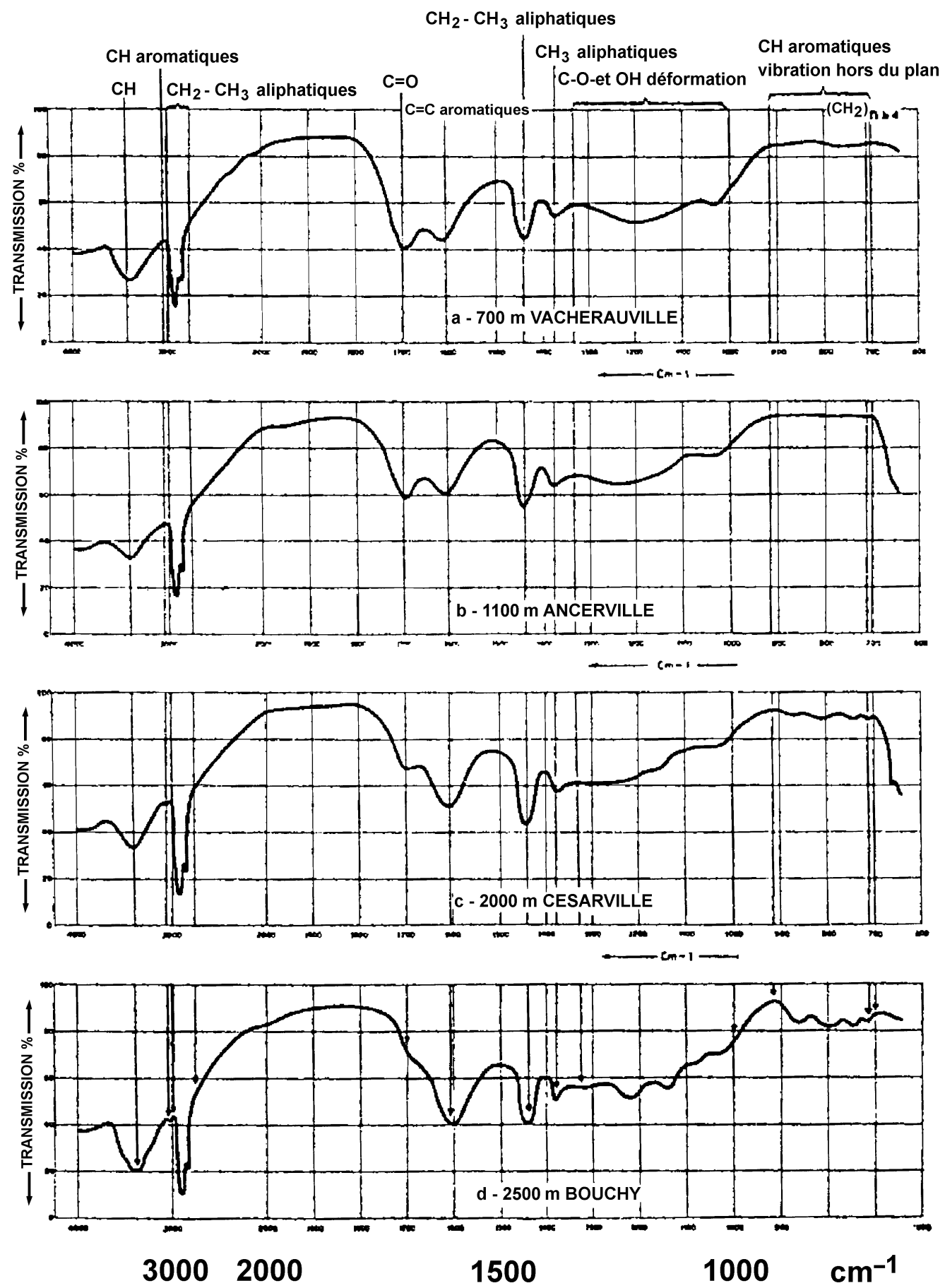

Fig. 3. Spectral evolution of kerogens from the same basin but different depths, from 700 to 2500 m. Adapted from Espitalie et al. (1973).

to the GC feature (see Figs. 2 and 3). Thus kerogen may partially contribute in this range, too.

Among the other bands, the $3.4-\mu \mathrm{m}$ band is prominent like in young kerogens (high $O / C$ and $H / C$ ratios), and its location and profile are pretty much the same in both cases (see Figs. 5a and 6a below). Also, the residual aromatic stretch near $3.3 \mu \mathrm{m}$ is very weak in both.

With their highest resolution instrument pointed towards Sgr A (IRS 3), Tielens et al. (1996) detected and assigned bands at 5.5, 5.8,6.1, 6.8 and predicted one near
$7.3 \mu \mathrm{m}$. The $5.5-\mu \mathrm{m}$ band is weak and was not confirmed by later observations (Schutte et al. 1998). The latter four bands are clearly present in young kerogens and have been given the same assignments. The aliphatic band at $6.8 \mu \mathrm{m}$ has $\tau=0.16$ and $\Delta \nu=20 \mathrm{~cm}^{-1}$, whose product is $3.2 \mathrm{~cm}^{-1}$ (Tielens et al. 1996). From Pendleton et al. (1994, Table 2 and Fig. 4), the 3.4- $\mu \mathrm{m}$ band has $\tau=0.23$ and $\Delta \nu=120 \mathrm{~cm}^{-1}$, the product of which is $28 \mathrm{~cm}^{-1}$. The ratio of integrated intensities is $\sim 9$, nearly the same as for kerogens (see above). 


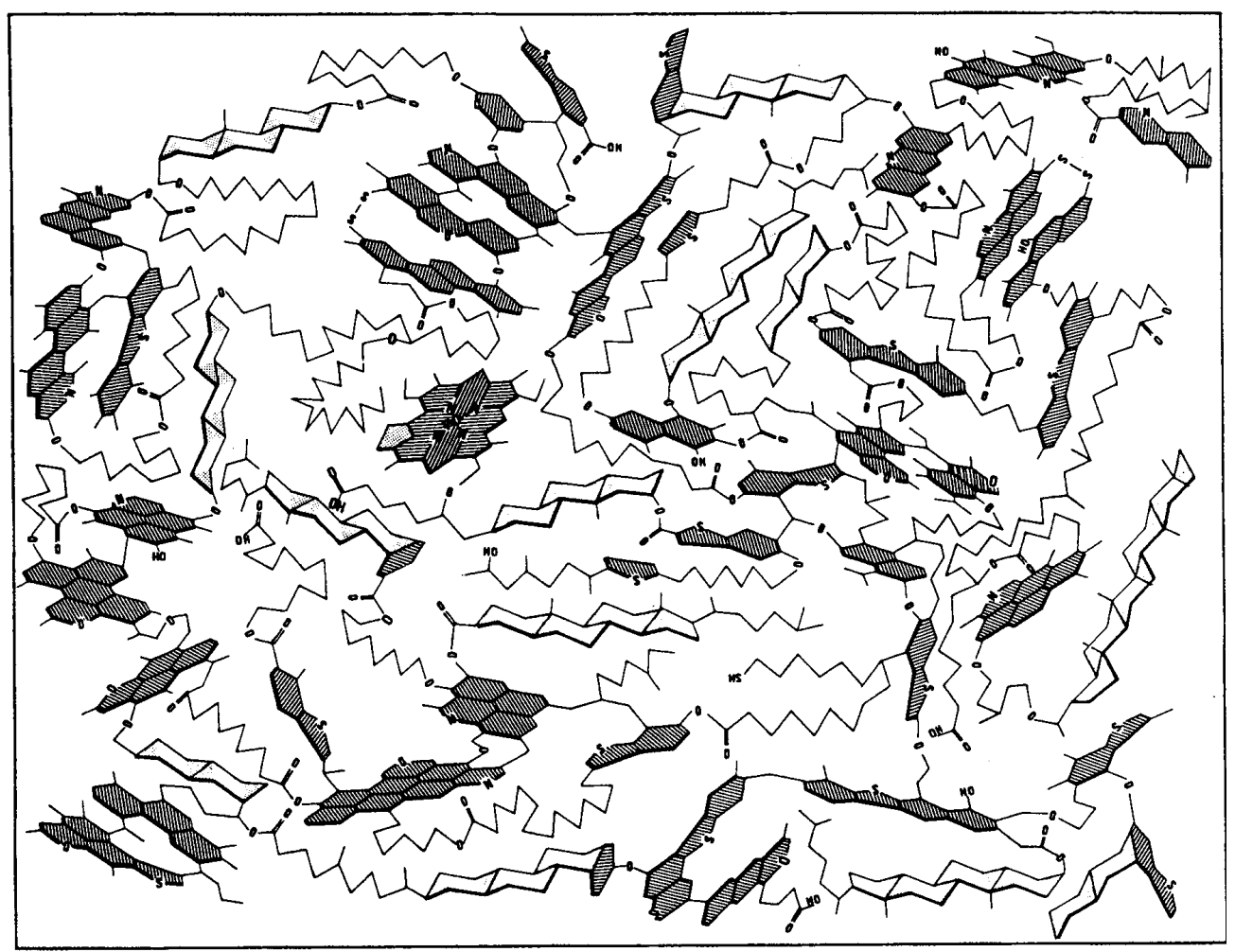

Fig. 4. Chemical representation of a type II kerogen at the start of diagenesis (adapted from Behar \& Vandenbroucke 1986). Following common practice, C-H bonds are not represented. Carbon chain skeletons are shown as broken, undulating, lines. The aromatic clusters of benzenic rings are shaded. Various functional groups and oxygen bridges are labeled.

In young kerogens of a given type, the $\mathrm{H}$ content is relatively constant, so the shape and strength of the $3.4-\mu \mathrm{m}$ blend do not depend very much on the evolutionary stage. By contrast, the relative intensities of the mid-IR are quite sensitive to evolution because of early loss of $\mathrm{O}$ atoms. This is evident, for instance, from Durand (1980, Chap. 6) and Espitalie et al. (1973). This indicator can therefore be used to choose a kerogen model carrier for a given astronomical spectrum. A visual inspection of the spectra in Espitalie et al., reproduced in our Fig. 3, suggests that the spectrum of sample c (type II, depth $2000 \mathrm{~m}$ ) comes closest to Tielens et al.'s Fig. 2 (1996). Note also in sample c the presence of an underlying plateau extending from 5 to $10 \mu \mathrm{m}$, which is welcome in view of the GC extinction curve obtained by Lutz (1999, Fig. 5), from the ISO satellite observations: the kerogen plateau is apt to fill the well-known dip exhibited by the silicate dust absorption in the same spectral range.

Sample c has $O / C=0.067$ and $H / C=1.24$; it is, therefore, closest to model IIb in the array of models provided by Behar \& Vandenbroucke (1986; see Sect. 2 above). Our Fig. 4 reproduces the corresponding chemical structure given by these authors. From their Tables 2 and 3, one can also deduce quantitative structural data such as:

- the relative concentration of aliphatic, naphtenic (in linear chains of benzenic rings) and aromatic carbon sites are, respectively, $\sim 45,14$ and $41 \%$;

- the average size of aromatic clusters is $\sim 3$ benzenic rings;
- the largest fractions of $\mathrm{O}$ atoms are engaged in ester $(-\mathrm{O}-\mathrm{C}=\mathrm{O})$ and ether $(\mathrm{C}-\mathrm{O}-\mathrm{C})$ groups; about 10 percent are in hydroxyl $(-\mathrm{OH})$ groups and 10 percent in 6membered rings and quinones;

- aliphatic chains $\left.\left(-\mathrm{CH}_{2}-\right)_{n}\right)$ are no longer than $n \sim 10$;

- according to Fig. 8 of Robin et al. (1975), $H($ methyl $) / H($ aliph $) \sim 0.2-0.4$.

Small molecules, such as $\mathrm{H}_{2} \mathrm{O}$ are trapped in the array by steric constraints; through H-bonding, they contribute to the inhomogeneous broadening of IR bands.

Finally, the integrated absorbance at $3.4 \mu \mathrm{m}$ for the model chosen above is $K=60 \mathrm{~cm} / \mathrm{mg}$ (Robin et al. 1975 ) and, for a bandwidth of $120 \mathrm{~cm}^{-1}$, the corresponding cross-section is $2.9 \times 10^{-20} \mathrm{~cm}^{2}$ per $\mathrm{C}$ atom.

\section{Inception and evolution}

Kerogen-like materials are bound to evolve, the faster the higher the ambient temperature (see Sect. 2). One cannot, therefore, be content with the comparison of one kerogen model with one particular line of sight. Fortunately, there are enough data from both sides to pursue the analogy. However, while the 3.4 feature is conspicuous in all kerogens (see Figs. 2 and 3), the open kerogen literature does not exhibit this feature on such a scale as to allow its sub-features to be quantitatively compared with the corresponding astronomical sub-features. The only exceptions to this are to be found in the astronomical literature cited in the introduction (Ehrenfreund et al. 1991, Fig. 4). 


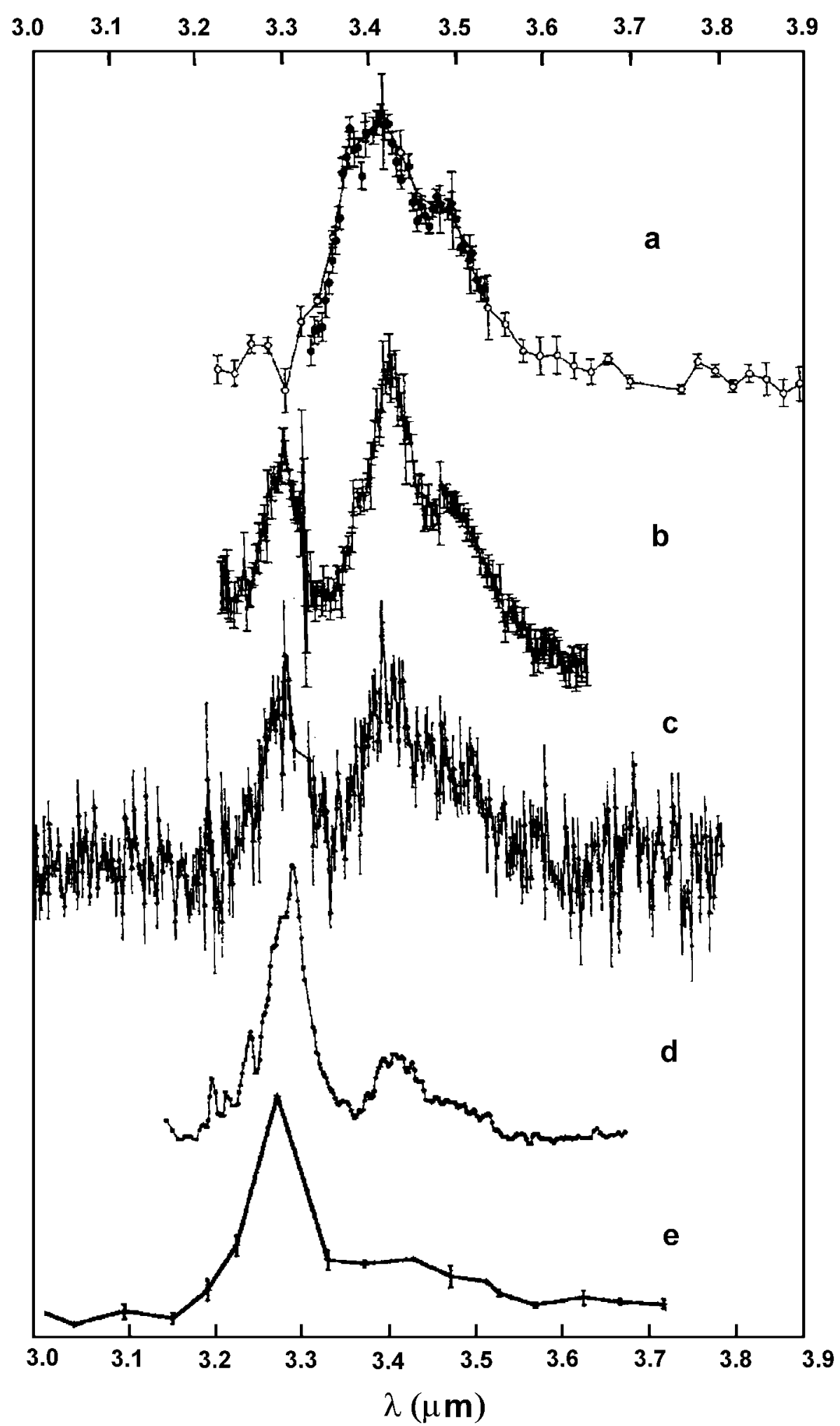

Fig. 5. The C-H stretch band of a) GC/IRS6E, in absorption (Pendleton et al. 1994); b-d) 3 post-AGB stars in emission: IRAS 08341+0852 (Joblin et al. 1996), IRAS 04296+3429 (Geballe et al. 1992) and CRL 2688 (Geballe et al. 1992); e) reflexion nebula NGC 2023 in emission (Sellgren 1984).

This is probably due to the fact that the $3.4-\mu \mathrm{m}$ blend had already been analyzed and discussed at length by coal experts (see e.g. Painter et al. 1981).
Moreover, there is a dearth of data on kerogens more evolved than $H / C \sim 0.5$. This is because kerogens are collected in sedimentary layers, which lay near the earth's 


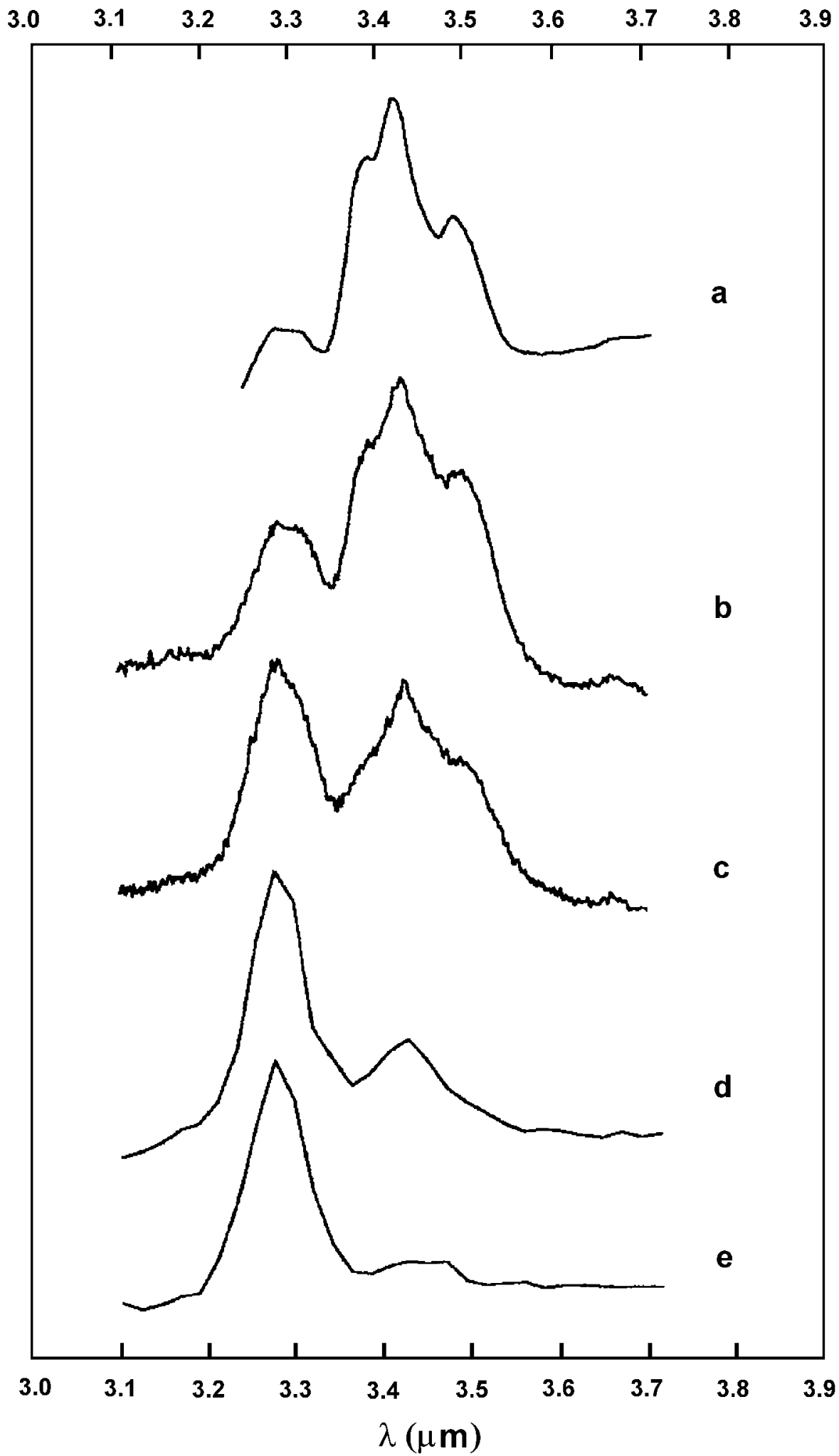

Fig. 6. The C-H stretch absorption band of coal as a function of evolutionary stage: a) Vouters mine $(O / C=0.06, H / C=0.75)$, b) Mericourt mine $(O / C=0.028, H / C=0.59)$, c) Escarpelle mine $(O / C=0.018, H / C=0.46)$, d) and e) Escarpelle sample annealed at 525 and $600 \mathrm{~K}$, respectively. Adapted from Guillois (1996).

surface and are relatively young. Also, the oil industry is only interested in the less evolved samples, which seem to be geographically associated with oil fields. On the other hand, there is plenty of data on coals from $H / C \sim 1$ down to 0 (semi-graphite), because coal has been searched for down to below $5000 \mathrm{~m}$ deep. Besides, there is a very rich 
collection of IR spectra of coals from various parts of the world. Now, kerogens and coals in evolved stages are very similar: they occupy the same quasi-vertical strip in the van Krevelen diagram (see Sect. 2). Thus, if one looks for a parallel between astronomical dust and natural carbonaceous materials, one can and should take advantage of both kerogen and coal data according to the needs of modelling.

Even so, some interpolation between available terrestrial materials was found to be necessary. This is made possible by the fact that heat treatment has long been known to mimic the natural evolution of coal and kerogen in the earth (see Papoular et al. 1996 for references). Figures 5 and 6 were constructed with the above in mind.

Figure 5 shows 5 spectra from different types of astronomical objects: a) the GC (IRS $6 \mathrm{E}$, in absorption); b-d) 3 post-AGB nebulae; e) a reflection nebula. The evolution is obvious and parallel to that in Fig. 6, which exhibits the spectra of 3 standard coals from progressively deeper mines (see Papoular et al. 1996): a) Vouters $(O / C=0.06, H / C=0.75)$, b) Mericourt $(O / C=0.028$, $H / C=0.59)$, c) Escarpelle $(O / C=0.018, H / C=0.46)$, and spectra of an Escarpelle sample annealed successively to $225^{\circ} \mathrm{C}(\mathrm{d})$ and $300{ }^{\circ} \mathrm{C}(\mathrm{e})$. The Vouters sample is the youngest coal and is akin to, but in a slightly more advanced evolutionary stage than, the model IIb kerogen chosen above. Its spectrum fits Fig. 5a better than do the kerogen samples in Fig. 4 of Ehrenfreund et al. (1991). The changes in the relative intensities of the aliphatic and aromatic stretch features clearly suggest a progressive aromatization of the carrier structure from top to bottom of Fig. 5.

In the case of Fig. 5c (IRAS $0.4296+3429$ ), the 11-13 $\mu \mathrm{m}$ range exhibits out-of-plane $\mathrm{C}-\mathrm{H}$ bend features which could be modelled, too, using the optical properties of the same (Escarpelle) coal as in Fig. 6c (Guillois et al. 1996). The same material was used successfully to model the near- and mid-IR spectra of two PPNe: IRAS $22272+5435$ and $07134+1005$. This indicates that dust present in the winds of AGB and post-AGB stars is already aromatic enough to exhibit a strong $3.3-\mu \mathrm{m}$ feature. The material in Fig. 6e is still more evolved and has lost most of its heteroatoms. The same can be expected for its counterpart in 5e, which must, therefore, have spent a very long time under photon irradiation in the DISM.

In novae, aromatization is much faster and nearly completed close to the dust birthplace, as, for instance, in Nova Cen 1986 (Hyland \& Mc Gregor 1988; Smith et al. 1994). This, of course, is due to the dust temperature being much higher than in the case of red giant or AGB stars.

Similarly, parallel evolutions occur in the mid-IR. For IS dust, this is illustrated by similar "transition" objects observed by Buss et al. (1990, 1993). In well-developed PNe (e.g. NGC 7027), the UIBs at 6.2, 7.7, 8.6 and $11.3 \mu \mathrm{m}$ stand out distinctly and are relatively narrow, according to the usual pattern in RNe. By contrast, the transition objects exhibit a much greater spectral variety, with the common characteristic of reduced visibility of the bands, which are blended in two prominent massifs peaking at about 8 and $12 \mu \mathrm{m}$, respectively (see, e.g., IRAS $22272+5435)$. This can be interpreted qualitatively by comparison with kerogen spectra (see Fig. 3 and assignments in Sect. 2) and coal spectra (Guillois et al. 1996): in the transition stages, oxygen and aliphatic carbon sites are still abundant, and responsible for the strong 5.85, 6.9 and $6-10-\mu \mathrm{m}$ features; the aromatic clusters are still small, allowing many polysubstitutions around the rings and, hence, many overlapping out-of-plane $\mathrm{C}-\mathrm{H}$ vibrations. The loss of heteroatoms upon ageing "purifies" the spectrum.

Goto et al. (2000) have recently produced a similar parallelism between near-IR astronomical spectra and spectra of QCC, using thermal processing of the initially aliphatic material up to $873 \mathrm{~K}$. However, they did not discuss the fingerprint region (mid-IR), which is so sensitive to oxygen content (see Figs. 2 and 3). But Sakata et al. (1987) have previously shown that unoxidized QCC is unable to mimic mid-IR celestial spectra correctly.

The structural changes which accompany these spectral changes are vividly illustrated in the sketches of the type shown in our Fig. 4, produced by Behar \& Vandenbroucke (1986).

In the framework of the present model, the absence of the usual carbonaceous features in red giant envelopes, their appearance in post-AGB circumstellar shells and the progressive strengthening of aromatic features and weakening of aliphatic ones through the PPN stage to the PN stage can only mean that, at the end of the PNe stage, the dust born in AGBs is already very much processed and almost as aromatic as the carrier of the UIBs (Unidentified IR Bands) observed in reflexion nebulae.

This scenario raises a number of issues. First, how is it that such an unevolved (aliphatic) dust as in Fig. 5a is observed towards the GC, seeming to imply that IS dust is mainly aliphatic? Indeed, if this dust were entirely distributed in the DISM, the fraction of the total available carbon, $C_{\text {avail }}$, that should have to be locked into it would be

$C_{\text {dust }} / C_{\text {avail }}=1.7 \times 10^{-2} \frac{H_{\text {avail }}}{C_{\text {avail }}} \frac{\tau(3.4)}{A_{\mathrm{v}}}$

using the cross-section per atom deduced from Sect. 4 $\left(2.9 \times 10^{-20} \mathrm{~cm}^{2}\right.$ per $\mathrm{C}$ atom $)$ and $N(H)=2 \times 10^{21} A_{\mathrm{v}}$; here, $(C / H)$ avail represents the relative number of carbon atoms available for dust, and lies between 80 and 200 per million, depending on authors. Now, according to Pendleton et al. (1994), $A_{\mathrm{v}} / \tau(3.4) \sim 150$ towards the GC. The locked fraction then turns out to lie between 0.6 and 1.4. This is hardly available, especially in view of the fact that relevant kerogen-like material is not aromatic enough to exhibit the right extinction in the visible/UV (see Khare et al. 1989) and should be complemented with some other carbonaceous dust. The same conclusion was reached on the basis of HAC models (Duley et al. 1998; Furton et al. 1999). Thus, only part of the feature intensity can be ascribed to dust in the DISM and in molecular clouds. Based on $A_{\mathrm{v}} / \tau(3.4) \sim 150$ towards the $\mathrm{CG}$ and 
$\sim 270$ in the local ISM, this fraction may rougly be estimated at $1 / 2$. The rest is likely to reside in the CS shells of the very abundant population of AGB stars revealed by the IRAS satellite towards the GC, as is the case for CRL618 (Lequeux \& de Muizon 1990).

This conclusion may seem to be at variance with the non-detection of the 3.4 feature in nearby AGB stars, either in emission or in absorption. Among others, Chiar et al. (1998) and Goto et al. (2000) also faced this paradox in the framework of their dust models and scenarios. Goto et al. suggested that an adequate chemical mechanism is suddenly switched on at the very end of the AGB phase. However, since carbon dust in some form clearly condenses even before the AGB phase, and in presence of abundant hydrogen, it seems less far fetched to speculate, on the ground of simple radiative theory, that, there and then, the dust temperature, density and distance from the star are such as to prevent the feature from showing up against the strong star light background. This would be in line with the very low contrast of the 3.3 and 3.4 features observed in the spectra of PPNe but not in those of PNe. Admittedly, however, this has yet to be worked out quantitatively.

Again, if the above implies that an important part of the IS carbonaceous dust is aromatic, then why is the 3.3$\mu \mathrm{m}$ band hardly seen in absorption through the DISM (see Schutte et al. 1998; Sellgren 2001), by contrast with the high visibility of the $217 \mathrm{~nm}$ band which is also assigned to aromatic material? This may be due to a combination of two factors: a) the intrinsic intensity of this band is so weak that it could not be measured accurately in kerogens; Wexler (1965) puts it at about $1 / 10$ of the aliphatic one; b) for the model material to finally exhibit the very aromatic UIB spectrum of reflection nebulae, $H / C$ must have fallen to about 0.3 , i.e. less than $1 / 4$ of the value used above to model the GC $3.4-\mu \mathrm{m}$ band.

Finally, both aliphatic and aromatic dusts expelled from CS shells are likely to ultimately settle in dense molecular clouds. How is it, then, that the carbonaceous dust of young stellar objects and dense molecular clouds is so different from that of the GC (Brooke et al. 1999, 1996)? Of course, any refractory dust inside the cloud is bound to be coated with ice. Pendleton (1999) considered the likelihood of the 3.4 feature being hidden by such a coating, but discarded it on the basis of laboratory experiments by Baratta \& Strazzulla (1990). Another solution was then proposed by Munoz Caro et al. (2001) and Mennella et al. (2001): they speculate that, somehow, there are enough FAR UV photons and/or cosmic rays inside the cloud for the composite grains to be processed in such a way as to dehyrogenate the carbonaceous component. At the same time, they fully acknowledge the need for theoretical modelling to validate their scenario.

At this point, it may be noted that the astronomical observations of dense clouds do not imply a complete suppression of signatures in the $3.4-\mu \mathrm{m}$ range: a broad $3.47-$ $\mu \mathrm{m}$ feature is still clearly detected along many sight lines; its red wing even seems to be nearly coincident with that of the diffuse ISM feature (Pendleton 1999). Now, Baratta \& Strazzulla's experiments show that a layer $0.1 \mu \mathrm{m}$ thick of water ice over an aliphatic organic residue considerably attenuates the 3.4 feature with respect to the ice feature, the former still peaks near $3.4 \mu \mathrm{m}$ and has only a shoulder near $3.5 \mu \mathrm{m}$. However, one may speculate that grains in dense clouds do not grow in distinct chemical layers. Another extreme case would be a thoroughly mixed combination of refractory and ice. Because of the very strong dielectric constant of water, the IR spectrum of such a mixture cannot be considered as the sum of the component spectra: the aliphatic feature is likely to be perturbed in shape and position in a non-trivial way. This has to be measured or be computed using, for instance, Maxwell Garnet's theory. Pending such developments, the issue remains open.

The emerging overall picture is that the abundant $\mathrm{H}$ and $\mathrm{O}$ atoms present in the envelopes of extreme C-stars and C-rich AGB stars favour the formation of highly aliphatic kerogen-like dust (cf. Fig. 5a). Afterwards, in the envelopes of PNe, the temperature and/or integrated photon radiation dose are high enough that aromatisation can nearly be completed (cf. Fig. 5e). However, some of the early dust is liable to escape into the ISM before this is indeed the case. Both aliphatic and aromatic dusts can therefore be observed in the ISM without implying that they are formed there.

More generally, the whole pattern of spectral evolution and diversity is less easily understood in terms of different specific, small, molecules (which cannot evolve spontaneously and continuously) than in terms of a family of materials like kerogens which differ continuously by the relative abundances of a small number of different classes of functional groups attached to a more or less aliphatic skeleton. Moreover, in the latter model, evolution does not require extreme conditions such as shocks, very low or high temperatures, nor high energy photons; ionization is not likely to make a big spectral difference.

\section{Conjectures and tests}

The kerogen model also helps us surmise the ultimate fate of carbonaceous dust. While the graphite found in the soil is the final outcome of kerogen/coal evolution, not all of these end up in graphite. Other conditions are necessary for this to be the case (see Bustin et al. 1995): first, a very high anisotropic pressure, as is known to apply very deep in Earth's crust; second, the precursor material must not be O-rich, for this would block early formation of large clusters of pericondensed aromatic rings, thus hindering "molecular orientation" and precluding the formation of graphite crystals (see Papoular et al. 1996). If, as in space, both conditions are not met, the end point of evolution is still $H / C=O / C=0$ but in "polygranular (or so-called semi-)graphite". Should this be the case for IS dust, as predicted by the present model, it would at least be compatible with the attested inability of crystalline graphite to reproduce the $217-\mathrm{nm}$ feature independent of grain size. 
This immediately suggests a simple experiment: take a sample of type II kerogen, anneal it up to $500 \mathrm{~K}$ for a few days, then make a VUV/vis spectrum and compare with the IS extinction curve.

Another test of the kerogen conjecture could be made at the other end of the spectrum, in the far IR. If kerogen is a valid model of dust, it should also exhibit the three conspicuous bands observed by Hrivnak et al. (2000), towards transient objects, PPNe and PNe, at nominal wavelengths 20, 26 and $30 \mu \mathrm{m}$. These features are weak and the measurement will not be easy, but an encouraging omen is that numerical models indeed exhibit these features and ascribe them to $\mathrm{OH}, \mathrm{NH}$ and -O- groups attached to mainly nonaromatic skeletons, like in kerogens (Papoular 2000).

Acknowledgements. Thanks are due to Dr. Vandenbroucke, Professor Rouxhet and Dr. Robin for interviews, letters, spectra and papers on kerogens.

\section{References}

Adamson, A., Whittet, D., Chrysostomou, A., et al. 1999, ApJ, 512,224

Baratta, G., \& Strazzulla, G. 1990, A\&A, 240, 429

Behar, F., \& Vandenbroucke, M. 1986, Rev. Inst. Fr. Petrole, 41(2), 173

Brooke, T., Sellgren, K., \& Smith, R. 1996, ApJ, 459, 209

Brooke, T., Sellgren, K., \& Geballe, T. 1999, ApJ, 517, 883

Buss, R., Cohen, M., Tielens, A., et al. 1990, ApJ, 365, L23

Buss, R., Tielens, A., Cohen, M., et al. 1993, ApJ, 415, 250

Bustin, R., Rouzaud, J.-M., \& Ross, J. 1995, Carbon, 33, 679

Chiar, J., Pendleton, Y., Geballe, T., et al. 1998, ApJ, 507, 281

Chiar, J., Tielens, A., Whittet, D., et al. 2000, ApJ, 537, 749

Duley, W., Scott, A., Seahra, S., et al. 1998, ApJ, 503, L183

Durand, B. 1980, Kerogen (ed. Technip, Paris)

Ehrenfreund, P., Robert, F., d'Hendecourt, L., \& Behar, F. 1991, A\&A, 252, 712

Espitalie, J, Durand, B., Roussel, J.-C., et al. 1973, Rev. Inst. Fr. Petrole, 28(1), 37

Furton, D., Laiho, J., \& Witt, A. 1999, ApJ, 526, 752

Geballe, T., Tielens, A., Kwok, S., \& Hrivnak, B. 1992, ApJ, 387, L29

Gibb, E., Whittet, D., Schutte, W., et al. 2000, ApJ, 536, 347

Goto, M., Maihara, H., Terada, H., et al. 2000, A\&AS, 141, 149

Greenberg, J. M. 1978, in Cosmic Dust, ed. J. A. M. Mc Donnell (New York, Wiley), 187

Guillois, O., Nenner, I., Papoular, R., \& Reynaud, C. 1996, ApJ, 464, 810

Guillois, O. 1996, Ph.D. Thesis, Paris-Sud University

Hrivnak, B., Volk, K., \& Kwok, S. 2000, ApJ, 535, 275
Hyland, A., \& Mc Gregor, P. 1988, IAU 135, NASA CP 3036, 101

Joblin, C., Tielens, A., Allamandola, L., \& Geballe, T. 1996, ApJ, 458, 610

Kerridge, J. 1990, in Carbon in the Galaxy, ed. J. Tarter, et al., NASA (Scient. Tech. Inform. Div.)

Khare, B., Thompson, W., Sagan, C., et al. 1989, in 1st Int. Conf. Lab. Res. Planetary Atmospheres, Bowri State U., MD

Kissel, J., Brownlee, D., Buchler, K., et al. 1986, Nature, 321, 280 and 336

Kwok, S., Volk, K., \& Hrivnak, B. 1999, A\&A, 350, L35

Leger, A., Gauthier, S., Defourneau, D., et al. 1983, A\&A, 117, 164

Lequeux, J., \& Jourdin de Muizon, M. 1990, A\&A, 240, L19

Lutz, D. 1999, in The Universe as seen by ISO, ESA SP-427, 623

Mennella, V., Munoz Caro, G., Ruiterkamp, R., et al. 2001, A\&A, 367, 355

Munoz Caro, G., Ruiterkamp, R., Schutte, W., et al. 2001, A\&A, 367, 347

Painter, P., Snyder, R., Starsinic, M., et al. 1981, Appl. Spectr., 35,475

Papoular, R., Conard, J., Guillois, G., et al. 1996, A\&A, 315, 222

Papoular, R. 2000, A\&A, 362, L9

Pendleton, Y., Sandford, S., Allamandola, L., et al. 1994, ApJ, 437,683

Pendleton, Y. 1999, in Solid interstellar matter: the ISO revolution, ed. L. d'Hendecourt, C. Joblin, \& A. Jones (EDP Sciences, Paris), 120

Robin, P., \& Rouxhet, P. 1976, Rev. Inst. Fr. Petrole, 31(6), 955

Robin, P., Rouxhet, P., \& Durand, B. 1975, Adv. Org. Geochem., ed. R. Campos, \& J. Goui (Enadisma, Madrid)

Sakata, A., Wada, S., Onaka, T., \& Tokunaga, A. 1987, ApJ, 320, L63

Sandford, S., Allamandola, L., Tielens, A., et al. 1991, ApJ, 371,607

Schutte, W., van der Hucht, Whittet, D., et al. 1998, A\&A, 337,261

Sellgren, K. 1984, ApJ, 277, 623

Sellgren, K. 2001, Spectrochim. Acta, 57A, 627

Smith, C., Aitken, D., \& Roche, P. 1994, Mon. Not. Roy. Soc., 267,225

Tielens, A., Wooden, D., Allamandola, L., et al. 1996, ApJ, 461,210

Unsworth, J., Fowler, C., Heard, N., et al. 1988, Fuel, 67, 1111

Van Krevelen, D. 1993, Coal (Elsevier, Amsterdam)

Volk, K., Kwok, S., Hrivnak, B., \& Szcerba, R. 2001, ApJ, submitted

Wada, S., Sakata, A., \& Tokunaga, A. 1991, ApJ, 375, L17

Wexler, A. 1965, Spectrochim. Acta, 21, 1735

Wexler, A. 1968, Appl. Spectr. Rev., 1, 80 